\title{
Integration and Efficient Lookup of Compressed XML Accessibility Maps
}

\author{
Mingfei Jiang and Ada Wai-Chee Fu, Member, IEEE
}

\begin{abstract}
XML}$ is emerging as a useful platform-independent data representation language. As more and more $\mathrm{XML}$ data is shared across data sources, it becomes important to consider the issue of XML access control. One promising approach to store the accessibility information is based on the CAM (Compressed Accessibility Map). We make two advancements in this direction: 1) Previous work suggests that for each user group and each operation type, a different CAM is built. We observe that the performance and storage requirements can be further improved by combining multiple CAMs into an ICAM (Integrated CAM). We explore this possibility and propose an integration mechanism. 2) If the change in structure of the XML data is not frequent, we suggest an efficient lookup method, which can be applied to CAMs or ICAMs, with a much lower time complexity compared to the previous approach. We show by experiments the effectiveness of our approach.
\end{abstract}

Index Terms-XML security, ICAM, CAM , XML accessibility lookup.

\section{INTRODUCTION}

W ITH more and more data represented in XML, the issue of controlled access for XML data has become extremely important. Encryption and digital signature methods, mainly used in electronic transactions, do protect the XML data in some aspects [4]. However, the design of a sophisticated access control mechanism for XML data still remains an open issue [15]. The models proposed by [3], [7] permit access control at different granularity levels and provide both positive and negative authorizations to better handle exceptions.

The study of access control for relational and objectoriented database models has a long history [5], [22], [10], [13], [14], [24], [20]. The access control for XML data is more complicated due to its semistructured feature. References [8], [3], [15], [19] are important work concerning the definition of various access control models for XML data. Fundulaki and Marx [12] survey some of these models and proposes a simple and unambiguous language to specify the access authorizations for XML data with XPath. While these efforts focus on constructing a semantically complete model to specify authorizations, but discuss little about the efficiency of space and evaluation time of a query in the presence of a set of authorizations. Fan et al. [11] is one possible complementary work of these efforts. It inherits the access control mechanism from relational database systems. For each user or group of users, a virtual security view is generated, which is embedded into the query before evaluating the query against the document. The method in [11] requires that the XML document conform to a DTD and every query be rewritten before being evaluated. Yet, another possible solution is to evaluate the authorizations in advance and generate an accessibility map for each user or a group of users, then to look up this map to discover the

- The authors are with the Department of Computer Science and Engineering, The Chinese University of Hong Kong, Hong Kong, China. E-mail:\{mfjiang, adafu\}@cse.cuhk.edu.hk.

Manuscript received 6 Jan. 2004; revised 9 Aug. 2004; accepted 2 Dec. 2004; published online 18 May 2005

For information on obtaining reprints of this article, please send e-mail to: tkde@computer.org, and reference IEEECS Log Number TKDE-0004-0104. accessible nodes for an incoming query. But, since the accessibility of every node is explicitly defined, accessibility lookup involves scanning the whole map, which can be highly inefficient in both space and time.

This paper studies the problem of compressing the accessibility map while still achieving acceptable lookup time. We assume that the authorizations have been clearly defined by one of the models, such as [12], and we evaluate them in advance to generate an accessibility map for each user group. Then, we try to compress these maps. The idea of the compressed accessibility map (CAM) is proposed by $\mathrm{Yu}$ et al. [25]. Efficient lookup on the compressed map is studied in [17]. However, in [25], one CAM is constructed for each possible operation type and for each user group. We notice that if we merge the CAMs for different types of operations into one integrated CAM (ICAM), we can further reduce the space needed for the accessibility maps. In addition, with the integrated CAM, if a user needs to look up the accessibilities for multiple types of operations for a data object, he/she needs to refer to only one ICAM, instead of multiple CAMs. We also study the lookup mechanism and propose a new method whose time complexity is much lower than that of the previous approach. The new method can also be applied to CAMs.

Our key contributions are as follows:

- We propose an algorithm to merge the optimal compressed accessibility maps for different types of operations into one ICAM. The result is a more compressed form of the accessibility maps which consolidate the information for each user/user group.

- We propose an efficient lookup method to determine the accessibility of a node with a time complexity of $O$ (depth of the requested node in the XML tree + log (the maximum fanout of the ICAM or (AM)). This method applies to both CAM and ICAM, and its time complexity compares favorably with the previously known time complexity of $O$ (depth of the requested node in $X M L$ tree $\times \log (C A M$ size $)$ ). 


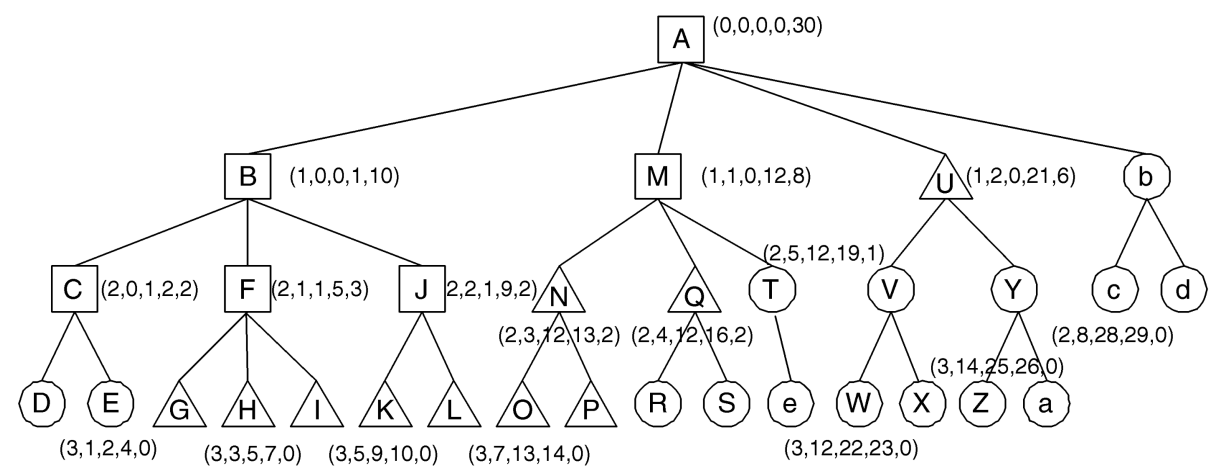

Fig. 1. A marked XML tree.

- We verify the effectiveness of the ICAM and the lookup method for both space and time requirements by experiments.

\section{CAM}

An XML document is one kind of semistructured data, which can be represented as an XML tree (or XML database tree in [25]) with a node for each element, attribute, and value in the document, and with an arc between each element and each of its subelements or attributes or values and between each attribute and its value(s) [1]. References [20] and [21] are the early works that describe a blueprint of the authorization mechanism for object-oriented and semistructured database. They denote an authorization by a 3-tuple, consisting of an authorization type (we refer to it as an operation type in this paper), an authorization object and a subject. The idea of implicit authorization is also introduced. That is, given a set of explicitly specified authorizations (authorization base), other authorizations, such as the operation type, object, and subject domains can be inferred. Authorization that is not stated explicitly and can be implied by authorization base is called implicit authorization. In this way, space can be saved by storing only the authorization base. Utilizing such an idea, the space for the accessibility map of an XML document can also be reduced.

CAM is one of the works that utilize the idea of implicit authorization along the dimension of authorization object. CAM stands for Compressed Accessibility Map. It is proposed by Yu et al. [25]. An accessibility map is generated by evaluating the authorizations against an XML document. It states the accessibility of each node in an XML document for a user. Storing the whole accessibility map takes up lots of space. In [25], the authors observe the structural locality of the accessibility of XML documents. That is, if a node is accessible (inaccessible) for some operation for a user, it is very likely that the same is true for its ancestors, its descendents, and its siblings. It becomes possible to record or label a node such that by default all the descendants are accessible (inaccessible), so that the labels of such descendants can be omitted to greatly reduce the number of labels. In other words, the accessibilities of some nodes can be induced by the labels of the others. As a result, instead of storing the whole accessibility map, it is possible to store only a small portion of the accessibility information. They refer to the resulting map as the compressed accessibility map, i.e., CAM.
It is convenient to first assume that when a node is accessible, then all of its ancestors are also accessible. In other words, the descendants of an inaccessible node must also be inaccessible. But, this assumption may not hold in reality. Yu et al. solve this problem by decomposing the whole tree into regions, each of which satisfies the assumption. They called such a region a unit region. The construction and lookup algorithms for unit regions are modified to handle the general case.

\subsection{First Step}

The first step of constructing a CAM in a unit region is to assign labels to the nodes in an XML tree of the form $(s *, d *)$, accordingly. Here, "*" can be either " + " or "-." $s+/ s-$ means that the current node is accessible/ inaccessible in terms of an operation type. ${ }^{1} d+/ d-$ means that the descendants of the current node are also accessible/ inaccessible unless they are overruled by the label of their closest ancestors. The notions of positive/negative/neutral nodes are proposed in [25] to determine the $d$ part of the internal nodes during the labeling step. An internal terminal node refers to a nonleaf accessible node with no accessible descendants. (An internal nonterminal node is a nonleaf node that is either inaccessible or that has some accessible descendants.)

Definition 1. [25] Given an operation, and an XML tree, a node $e$ is positivelnegative provided that $e$ is an accessible/ inaccessible leaf, or $e$ is an internal nonterminal node with more positive/negative than negative/positive children. A node $e$ is neutral if it is an internal nonterminal node with the same number of positive and negative children. ${ }^{2}$

Here, we give an example to illustrate the first step in the construction of a CAM in a unit region, the details of the algorithm can be found in [25].

Example 1. Fig. 1 shows an XML document in the form of a tree [1]. (Let us ignore the numerical labels in the tree for now.) The square nodes can be written and read, the triangular nodes can only be read, and the circular nodes are inaccessible. In order to construct a CAM for the read operation, we first assign labels to the nodes in the XML tree of the form $(s *, d *)$ according to the accessibilities for the read operation. The labeling is done bottom up:

1. When we say that a node is accessible for an operation, it means that the operation can be executed on the node, or the operation is permitted at the node.

2. Note that an internal terminal node is neither positive, nor negative, nor neutral. 


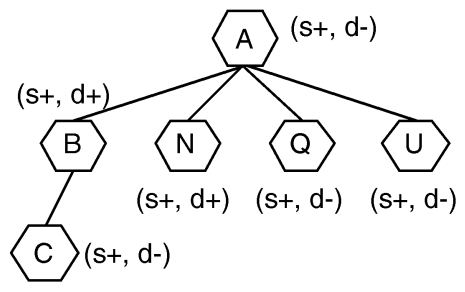

(a)

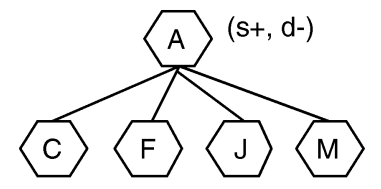

$(s+, d-)(s+, d-)(s+, d-)(s+, d-)$

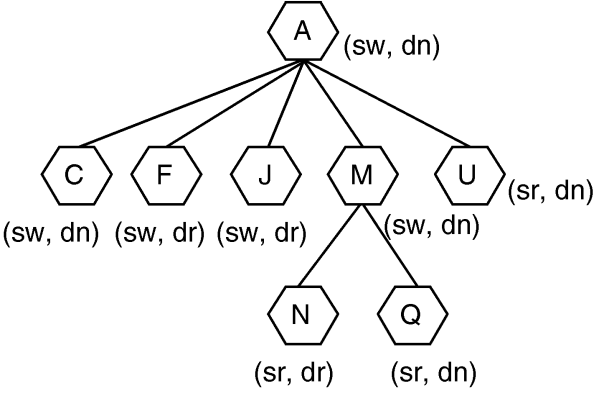

(c)

Fig. 2. CAMs and ICAM for the example XML tree.

1. $L_{r}(D)=L_{r}(E)=(s-, d-)$ (inaccessible and negative).

2. $L_{r}(C)=(s+, d-)$ (internal terminal).

3. All the nodes in the subtrees rooted at $F$ and $J$ are labeled with $(s+, d+)$ (accessible and positive).

4. $L_{r}(B)=(s+, d+)$ (accessible and positive).

5. All nodes in the subtree rooted at $N$ are labeled $(s+, d+)$ (accessible and positive).

6. $\quad L_{r}(R)=L_{r}(S)=(s-, d-) \quad$ (inaccessible and negative).

7. $L_{r}(Q)=(s+, d-)$ (internal terminal).

8. $\quad L_{r}(T)=L_{r}(e)=(s-, d-)$ (inaccessible and negative).

9. $L_{r}(M)$ is deferred, for it is neutral.

10. All nodes in the subtree rooted at $U$ (except $U$ ) are labeled $(s-, d-)$ (inaccessible and negative).

11. $L_{r}(U)=(s+, d-)$ (internal terminal).

12. All nodes in the subtree rooted at $b$ is labeled with $(s-, d-)$ (inaccessible and negative).

13. $A$ is neutral. As it is the root, we arbitrarily label it with $(s+, d-)$. It leads $M$ to be labeled as $(s+, d-)$, too.

\subsection{Second Step}

The second step of the construction is to delete redundant labels in a certain order. There are two kinds of redundant labels in a labeled XML tree [25]. 1) The subsumed labels. A subsumed label is a label that is not in the CAM but that can be induced by other labels in the CAM. 2) The upward redundant labels. For such a label, the " $\mathrm{s}$ " can be induced, and " $\mathrm{d}$ " is immaterial because all the children of that node are either labeled or upward redundant in the CAM.

Definition 2 (Upward Redundant Label [25]). A label of a node e in a CAM $C$ is said to be upward redundant if e has a accessible proper descendant, ${ }^{3}$ and for every child $c$ of $e$, either $c$ is labeled in $C$ or $c$ is upward redundant.

Example 2. We illustrate Step 2 of the construction by continuing with Example 1. In this step, we delete the redundant labels (subsumed labels are deleted before upward redundant labels, while the order of subsumed and upward redundant label deletion is immaterial), we obtain the CAM for the read operation, which is shown in Fig. 2a. For example, $L_{r}(D)$ and $L_{r}(E)$ are subsumed

3. We consider $e$ to be its own descendent, a descendant of $e$ is proper if it is not equal to $e$. by $d-$ of $L_{r}(C)$. Thus, they are deleted. There is no upward redundant label in this example. The CAM for the write operation is shown in Fig. $2 \mathrm{~b}$.

Later on, we refer to a CAM for an operation $x$ as $C A M_{x}$. The deleted labels for the XML tree can be induced from the labels in the CAM by Definition 3. For example, given $C A M_{r}$, the label of node $\mathrm{M}$ can be induced from the label of A and that of either $N$ or $Q$.

Definition 3. [25] Given a CAM for an operation $z, C A M_{z}$, the induced label at a node $e$ in the corresponding labeled database tree (we use the term XML tree in this paper), written $L_{z}(e)$, is its label in $C A M_{z}$ if one exists. Else, let $y$ be the nearest labeled ancestor of $e$ if any (note that $e$ is also considered its own ancestor and its own descendent):

- If y has a label $(s+, d+)$ and $L_{z}(e)=(s+, d+)$.

- If y has a label $(s-, d-)$ and $L_{z}(e)=(s-, d-)$.

- If y has a label $(s+, d-)$ :

- If e has a descendant labeled either $(s+, d-)$ or $(s+, d+), L_{z}(e)=(s+, d-)$.

- Else $L_{z}(e)=(s-, d-)$.

- If e has no labeled ancestor, ${ }^{4} L_{z}(e)=(s+, d-)$.

\section{INTEGRATED CAM (ICAM)}

\subsection{Motivation of ICAM}

In [25], if there are multiple operation types defined in the system, a different CAM is constructed for each type of operation and for each user group. We observe that the set of accessible nodes for different types of operations ${ }^{5}$ may overlap, e.g., as shown in Figs. $2 a$ and $2 b$, node $C$ is labeled in both CAMs for read and write operations. Assume that if the write operation is allowed, the read operation is automatically allowed. We say that write covers read. The "cover" relation of operations can be represented by an operation hierarchy in which a node "covers" its descendent nodes. With the assumption of such a hierarchy, it is possible to combine all the CAMs for different operations to

4. In this case, $L_{z}(e)$ is undefined in [25]. From the assumption that if a node is accessible, all its ancestors should be accessible, $e$ has no labeled ancestor only when it and all its ancestors are upward redundant. Then, they should have at least one accessible labeled descendant by the definition of the upward redundant label. Hence, $e$ should be accessible, implying that the "s" part of $L_{z}(e)$ must be "+." While the " $\mathrm{d}$ " part of its label is not essential for all of its children are labeled or upward redundant. We assign "-" to it in order to simplify our proof.

5. In the remaining discussions, we shall refer to "type of operation" simply as "operation," if no ambiguity may arise. 


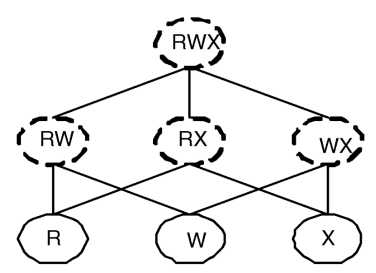

(a)

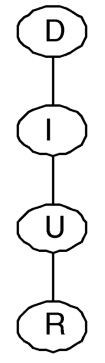

(b)

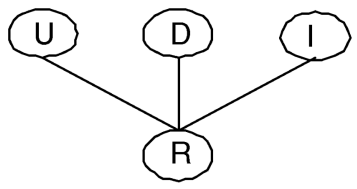

(c)

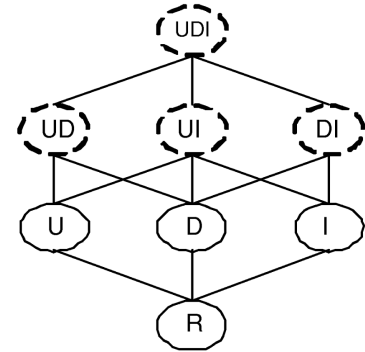

(d)

Fig. 3. Operation hierarchies (the composite operations are represented in the dashed circle).

form an integrated CAM (ICAM). Given an XML tree, an ICAM is a compressed accessibility map where labels of a subset of the XML nodes are recorded, the accessibility information of all operations have been integrated at each labeled node, and from this map, the accessibility of each node in the XML tree for each operation can be determined. If we use a label having a form of $(s *, d *)$ to represent the accessibility information of each node in an ICAM, the label can be obtained from merging the labels in the CAMs for each operation. Roughly speaking, in this example, we label a node with $(s *, d *)$ where $*$ can be $r, w, n$. The label $s n$ means that the node is not accessible, $s r$ means it is read accessible, $s w$ means it is write accessible, which implies it is also read accessible. The $d$ part can be understood similarly for the descendants of the current node. For example, Fig. 2c shows an ICAM for the read and write operations of the XML tree in Fig. 1. We will further explain the meanings of the labels in the following sections.

In practice, many authorization mechanisms, for example, [20], [3], [6], assume an operation hierarchy, in which a partial ordering of operations exists, so that if a user is allowed to do one operation, some other operations are automatically permitted. If no explicit hierarchy exists, there is always an implicit hierarchy for the multiple operations, which is composed of atomic and composite operations. For example, the Unix file system supports three atomic operations: read, write, and execute $(r, w, x)$. An implicit operation hierarchy exists, which contains four composite operations: ReadWrite (rw), ReadExecute $(r x)$, WriteExecute $(w x)$, and ReadWriteExecute (rwx), as illustrated in Fig. 3a.

Thus, we claim that assuming an operation hierarchy is reasonable and beneficial. Under such an assumption, when the CAMs for different operations overlap in the node sets, the size of ICAM is smaller than the total size of the CAMs. Thus, we can further reduce the space needed to store the accessibility map. In the following sections, unless specified, the term "operation" refers to both atomic and composite operations.

\subsection{Operation Hierarchy}

Here, we introduce the operation hierarchy more formally, where every element in the hierarchy stands for an allowed operation. And, an operation may "cover" other operations.

Definition 4. Operation $x$ covers operation $y$ if whenever operation $x$ is permitted at a node, operation $y$ is also permitted at that node. We denote this by $x \geq y$. It defines a partial ordering on the operations.
We say that $x$ immediately covers $y$ if $x \geq y$ and there is no distinct $z$ with $x \geq z \geq y$. If $x \geq y$ and $x \neq y$, we can write $x>y$. If $x$ does not cover $y$ and $y$ does not cover $x$, we write $x \sim y$. A graph of the cover relation is the digraph whose vertices are the operations and which has an edge from $x$ to $y$ if and only if $x$ immediately covers $y$. If we always draw $x$ above $y$ when $x>y$, we have an operation hierarchy. We make the following assumption.

Assumption 1. Given any two operations, $x$ and $y$, in an operation hierarchy, where $x$ and $y$ have no "cover" relation, i.e., $x \sim y$, if it is possible that $x$ and $y$ are both allowed at a node in the XML tree, there should be a composite operation, $o$, s.t. o immediately covers both $x$ and $y$. And, when $x$ and $y$ are both permitted at a node, $o$ is permitted at the same time.

Then, the label of $o$ can be inferred from those of $x$ and $y$. That is, if both the $s$ part of $x$ and $y$ are ",+ " then the $s$ part of $o$ is " + "; otherwise, it is "-." The $d$ part of $o$ 's label can be inferred in the same way.

Fig. 3 shows three possible operation hierarchies for four atomic operations, Delete $(D), U$ pdate $(U)$, Insert $(I)$, and $\operatorname{Read}(R)$. In Fig. 3b, $D$ covers all the other three operations; $I$ covers $U$ and $R$; and, $U$ covers only $R$. In Fig. 3c, $D, U, I$ cover $R$, but they do not cover each other, and at most one of them is permitted at a time. For example, once a node can be deleted, it can be read because $D$ covers $R$. But, it cannot be updated or inserted. If more than one operations are allowed at a time, the operation hierarchy becomes Fig. 3d, in which one composite operation is created for each possible combination of atomic operations. And, these composite operations are presented in dash circles. An operation hierarchy has the following properties.

Property 1. Every operation hierarchy implies a virtual null operation, $n$, denoting no operation is permitted. $n$ is covered by all the other operations in the operation hierarchy. And, for every node, $e$, in the XML tree, $L_{n}(e)=(s+, d+)$.

Property 2. The operation hierarchy is a directed acyclic graph (DAG) and can be topologically sorted.

An operation hierarchy is constructed by the "cover" relation, which is asymmetric. Hence, the graph on this relation cannot have a cycle. Thus, the operation hierarchy is a DAG. A topological sort [2] of a digraph $G$ is a sequence of all the nodes of $G$ so that if $x$ appears before $y$ in the sequence, there is no path from $y$ to $x$ in $G$. It is proved by construction in [23, p. 549] that every DAG can be topologically sorted. Hence, Property 2 holds. For example, one of the topological sorting of the operation hierarchy in 
Fig. $3 \mathrm{~d}$ is $U D I \gg U D \gg U I \gg D I \gg U \gg D \gg I \gg R$. And, it is easy to see that given two operations, $x$ and $y$, in an operation hierarchy, $x \geq y$ implies $x \gg y$, but not vice versa. And, if $x \gg y$, we say that $x$ is larger than $y$, or $y$ is smaller than $x$.

It is obvious that the slimmer the operation hierarchy, the higher the probability that the CAMs will overlap, which leads to more space being saved. Even if there are no relationships among the atomic operations (see Fig. 3a), it is still possible for the CAMs to overlap. That is, space can still be saved by the ICAM.

\section{Construction and LOOKUP OF ICAM}

In this section, we will discuss the algorithm to construct and look up an ICAM efficiently.

\subsection{Node Labels}

The node label of the ICAM is an extension of that of the CAM. Recall that the label of a node, $e$, in $C A M_{x}$ is of the form $(s *, d *)$, where * can be "+" or "-." Let us refer to such a label as $L_{x}(e)$. We use $L(e)$ to denote the label of node $e$ in the integrated CAM (ICAM). It is of the form $(s x, d y)$, where $x$ and $y$ are operations in the given operation hierarchy. The first part, $s x$, determines that the current node is accessible for operation $x$ by the user. And, the second part, $d y$, denotes that the descendents of the current node are accessible for operation $y$ by the user unless overruled by the label of a closer labeled ancestor.

For example, in Fig. 2c, the label of node $F,(s w, d r)$, means that node $F$ is writable but its descendants can only be read unless overruled by a closer labeled ancestor.

It is possible that operation $x$ is denied at node $e$, but permitted at a descendant of node $e$. In order to cope with this general case, we extend the idea of the unit region from [25] to the multioperation case. That is, we decompose the tree into regions, such that within each region, for any operation $x$, if $x$ is permitted at node $e$ in the XML tree, it is also permitted at all the ancestors of $e$. We also call such a region a unit region. We first define the label merging rule and inducing rule based on one unit region. Then, we make some modifications to cope with the general case.

\subsection{ICAM Construction for a Unit Region}

In this section, we will describe the algorithm of constructing an ICAM in a unit region. There are many possible ICAMs, but we aim at building a minimal one. The algorithm to construct an ICAM consists of the following main steps:

1. For each atomic operation, label the XML tree according to the algorithm in [25], except that when encountering a neutral root, we choose a favorite label by Rule 1 (see below), instead of assigning a label arbitrarily. Construct CAMs for the atomic operations. However, in this step, we do not remove the redundant labels in the CAMs.

2. From the CAMs construct an ICAM.

Rule 1 (Favorite Label for the Neutral Root). Consider an $X M L$ tree and operations $x_{1}, x_{2}, \ldots, x_{n}$, and a topological sort $x_{1} \gg x_{2} \gg \ldots \gg x_{n}$, based on the cover relation. In the process of constructing $C A M_{x_{i}}$, if the root is neutral in terms of $x_{i}$, we label the root as follows: We construct CAMs in the topological order of the operations, from $x_{1}$ to $\left.x_{n}, 1\right)$ if $\exists x_{j}$, s.t. $x_{j}$ immediately covers $x_{i}$, let $L_{x_{i}}($ root $)=L_{x_{j}}($ root $)$ and 2) else $L_{x_{i}}($ root $)=(s+, d+)$.

We follow the procedure to construct $C A M_{x}$ for each operation $x$, except that we do not really delete the redundant labels for $x$, instead they are marked with " $\mathrm{D}$ " for "deleted." The CAMs are actually maintained by storing at each XML node a list of (label, delete_mark) pair, one pair for each operation, where label is the label for an operation and delete_mark is " $\mathrm{D}$ " or "Null." Then, all of the XML nodes whose labels are not all marked as " $\mathrm{D}$ " will form the nodes in the ICAM. We merge the labels of such nodes using the Label Merging Rule.

Rule 2 (Label Merging Rule (LMR)). Consider $n$ different operations, $x_{1}, x_{2}, \ldots, x_{n}$, and a topological sort of $x_{1} \gg x_{2} \gg$ $\ldots \gg x_{n}$ based on the cover relation. The integrated label of a node $e$ in the ICAM is $(s x, d y)$, where $x$ is the smallest operation covering all $x_{i} s$ such that $L_{x_{i}}(e)=(s+, d *)$, and $y$ is the smallest operation covering all $x_{j} s$ such that $L_{x_{j}}(e)=(s+, d+)$.

Note that if $X=x_{1}, x_{2}, \ldots x_{n}$ includes all operations in the corresponding operation hierarchy, then the minimal operation covering all $x_{i} \mathrm{~s}$ such that $L_{x_{i}}(e)=(s+, d *)$ must also be in set $X$ and it must have a label of $(s+, d *)$ for node $e$. Similarly, the minimal operation covering all $x_{j}$ s such that $L_{x_{j}}(e)=(s+, d+)$ must be in $X$ and have a label of $(s+, d+)$ for $e$. This is because if two or more operations are accessible at the node, then an operation must exist in the hierarchy which is the minimal operation covering these operations and it must also be accessible. Also due to this assumption, given a label $(s x, d y)$ for node $e$ in the ICAM, $x$ will not cover any operation, $x_{i}$, such that $L_{x_{i}}(e)=(s-, d-)$. And, $y$ will not cover any operation $x_{j}$, such that $L_{x_{j}}(e)=(s *, d-)$.

The label merging step may introduce new redundant labels. Since the labels in the ICAM are nonredundant in at least one CAM, the only possibility of redundant labeling in the ICAM is upward redundant labeling, whose definition is given in Definition 5 below. It was first introduced by [25], but multiple operations are considered here.

Definition 5 (Upward Redundant Label in an ICAM). A label at node $e$ in an ICAM $I$ is said to be upward redundant if e has an accessible proper descendant for each operation, and for every child $c$ of e, either $c$ is labeled in I or $c$ is upward redundant.

We remove the upward redundant labels while we merge the labels by traversing the XML tree in postorder. Note that for a CAM, only the upward redundant labels without any labeled proper ancestor are removed. That is because any node with upward redundant label and with labeled proper ancestors are subsumed and deleted when the subsumed labels are removed. However, for an ICAM, a node is labeled after the merging step by LMR if it is not subsumed in at least one CAM, thus when removing the upward redundant labels, those with labeled ancestors should also be considered. Fig. 4 describes the construction of a minimal ICAM. By a minimal ICAM, we refer to an ICAM with no redundant labeling due to subsumption or upward redundancy. Here, we given an example to show how a minimal ICAM is generated from CAMs by Algorithm 1. 


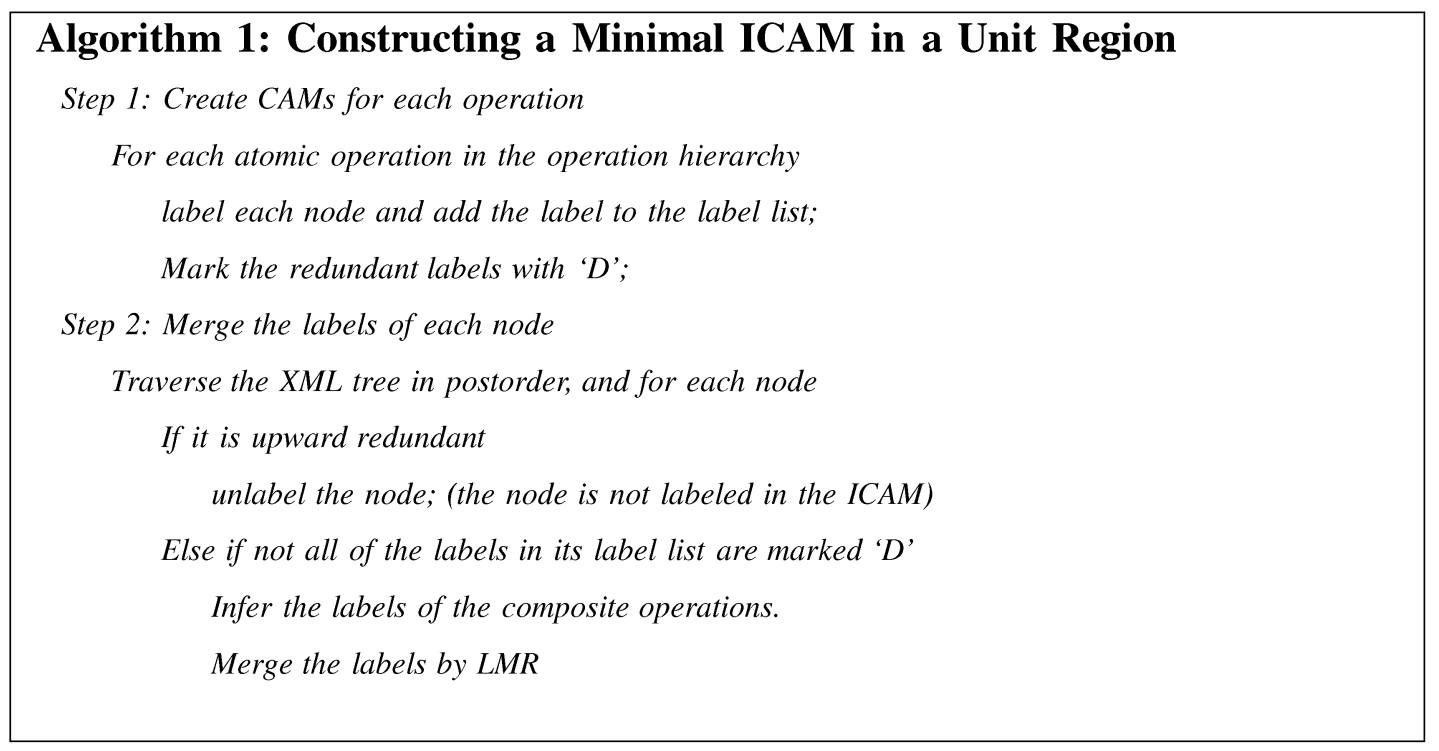

Fig. 4. Constructing a minimal ICAM in a unit region.

Example 3. Suppose there is an XML tree, as shown in Fig. 1, and the system supports two atomic operations, $r($ read $)$ and $w$ (write), where $w$ covers $r$. We also assume another virtual operation, $n$, which denotes that no operation is permitted at the node. The topological order of these three operations is: $w \gg r \gg n$. Then, there are two steps to construct an ICAM:

- Step 1: For each atomic operation, we first label the XML tree by $(s *, d *)$, where " $*$ " can be "+" or "-." Then, we mark the redundant labels. While $C A M_{r}$ is being constructed, a neutral root $(A)$ is encountered. According to Rule 1 , as $C A M_{w}$ is constructed first and $L_{w}(A)=(s+, d-)$ ( $A$ is accessible and negative for $w$. Note that $M$ is an internal terminal node for $w$ and is neither positive nor negative. Hence, $A$ has one neutral child $(B)$ and two negative children $(U$ and $b)$ ), we choose $(s+, d-)$ for $L_{r}(A)$. As a result, a labeled XML tree is obtained and each node in it maintains a label list. For operation $w$, all the labels except those of of $A, C, F, J$ and $M$ are marked "D." For operation $r$, all the labels except those of $A, B, C, N, Q$ and $U$ are marked "D."

- Step 2: For each node in the XML tree, if not all of its labels in the CAMs are marked " $\mathrm{D}$," we merge its labels for every operation. In this example, for node $A, L_{w}(A)=(s+, d-)$, and $L_{r}(A)=(s+, d-)$, thus $L(A)=(s w, d n)$. For node $F, L_{w}(F)=(s+, d-)$, and $L_{r}(F)=(s+, d+)$. Although $L_{r}(F)$ is marked " $\mathrm{D}$ " for operation $r, F$ has to be labeled in the ICAM, for it is not marked " $\mathrm{D}$ " for $w$. Then, according to LMR, $L(F)=(s w, d r)$. Labels of the other nodes can be obtained in the same way. During the label merging, we also find that node $B$ is upward redundant because $B$ has an accessible labeled descendant for both $w$ and $r$, and all its children are labeled. Thus, we delete the label of $B$. The resulting ICAM is shown in Fig. 2c.

In the construction, the time needed for Step 1 is the product of the number of atomic operations and the time for constructing a CAM for one operation. As the time for constructing a CAM is linear to the number of nodes in the XML tree, and the number of the atomic operations can be considered as a constant, the time complexity for Step 1 is $O$ (number of nodes in the XML tree). The time complexity of Step 2 is also the same. Hence, the overall time for constructing an ICAM is proportional to the number of nodes in the XML tree.

If we refer to the number of nodes in a CAM/ICAM as the size of the CAM/ICAM, then the following theorem holds.

Theorem 1. The size of ICAM is equal to or smaller than the total size of CAMs for different operations.

Proof. As shown in the algorithm, in the process of the label merging, we only consider any node which is not marked as redundant in at least one CAM. In other words, we only merge the labels of the nodes in the CAMs. Thus, if the labeled nodes in all CAMs are different, then the size of the ICAM is equal to the total size of the CAMs, otherwise the size of the ICAM is strictly smaller.

Theorem 2. Given an XML tree and atomic operations $x_{1}, x_{2}, \ldots, x_{n}, C_{x_{1}}, C_{x_{2}}, \ldots C_{x_{n}}$ are CAMs that label the root by Rule 1. $C_{x_{1}}^{\prime}, C_{x_{2}}^{\prime}, \ldots, C_{x_{n}}^{\prime}$ are CAMs constructed in the same way except that they may not follow Rule 1 . Then, the size of the ICAM generated from $C_{x_{1}}, C_{x_{2}}, \ldots, C_{x_{n}}$ is no larger than the one generated from $C_{x_{1}}^{\prime}, C_{x_{2}}^{\prime}, \ldots, C_{x_{n}}^{\prime}$.

Theorem 3. Given n optimal CAMs,

$$
C A M_{x_{1}}, C A M_{x_{2}}, \ldots, C A M_{x_{n}},
$$

the ICAM generated by Algorithm 1 is minimal (in the number of nodes) in that no redundant node remains.

The proofs of the above theorems are given in the full version of this paper in [18]. 


\subsection{ICAM Lookup in a Unit Region}

In this section, we describe the algorithm of looking up an ICAM in a unit region. The following lemma is essential for this purpose.

Lemma 1. In a unit region, if the integrated label of a node $e$ is $(s x, d y)$, then $x \geq y$.

Proof. Note that $x$ and $y$ are both permitted at $e$ (in a unit region, if $y$ is permitted at descendants of $e, y$ is also permitted at $e$ ). According to LMR, $x \geq y$, since $x$ is the minimal operation covering all operations permitted at $e$ in the CAMs.

Given a label in the ICAM, the label of a node in terms of an operation can be induced by the Label Inducing Rule.

Rule 3 (Label Inducing Rule (LIR)). Given an ICAM and $a$ node $e$ in the corresponding XML tree, the induced label $I L_{z}(e)$ for operation $z$ is determined according to the following rule:

1. If $e$ is labeled in the ICAM, and $L(e)=(s x, d y)$

- if $y \geq z$, then $I L_{z}(e)=(s+, d+)$,

- $\quad$ else if $x \geq z$, then $I L_{z}(e)=(s+, d-)$, and

- $\quad$ else $I L_{z}(e)=(s-, d-)$.

2. Else, suppose $f$ is the nearest labeled ancestor of $e$ in the ICAM if any, and $L(f)=(s x, d y)$

- if $y \geq z$, then $I L_{z}(e)=(s+, d+)$,

- $\quad$ else if $(x \geq z)$, then

if $e$ has a labeled descendant, $p$, in the ICAM, labeled $(s m, d n)$, and $(m \geq z)$, then $I L_{z}(e)=(s+, d-)$ and else $I L_{z}(e)=(s-, d-)$.

- $\quad$ else $I L_{z}(e)=(s-, d-)$.

3. Else if e has no labeled ancestor, $I L_{z}(e)=(s+, d-)$.

Example 4. Consider the ICAM in Fig. $2 \mathrm{c}$ as an example. Suppose $I L_{r}(F)$ is requested. Because $F$ is labeled in the ICAM, $L(F)=(s w, d r)$ and $r \geq r, I L_{r}(F)=(s+, d+)$ according to the $L I R$. If $I L_{r}(B)$ is requested, we should locate its nearest labeled ancestor first. It is $A$ and $L(A)=(s w, d n)$. Because $w \geq r$, but $\neg(n \geq r)$, we need to check if $B$ has any labeled descendant. We find $C$ and $L(C)=(s w, d n)$. So, we get $I L_{r}(B)=(s+, d-)$ by $L I R$.

Note that the $d$ part of $I L_{r}(B)$ and $L_{r}(B)$ are not equal. After the ICAM is constructed from $L M R$, and before the upward redundant labels are deleted, $B$ should be labeled in the ICAM. In this ICAM, $L(B)=(s w, d r)$. Then, $I L_{r}(B)=$ $(s+, d+)$ by $L I R$, which equals $L_{r}(B)$, as induced from $C A M_{r}$. When upward redundant labels are removed, $I L_{r}(B)$ becomes $(s+, d-)$. However, the $d$ part of such an induced label does not affect the correct accessibility deduction for the other nodes. This is because the children of an upward redundant node are either labeled or upward redundant in the ICAM. The following theorem, which is proven in [18], makes sure that LIR is correct.

Theorem 4. Suppose an ICAM, C, is generated by LMR for the operations $x_{1}, x_{2}, \ldots, x_{n}$, where upward redundant labels are not removed. Given a node e in the corresponding XML tree, its induced label for operation $x_{i}$ in $C$ is the same as its induced label in $C A M_{x_{i}}$.

After an induced label is obtained, the accessibility of the node is easily deduced, as in [25]. In particular, given a node $e$ and $I L_{z}(e)$, the accessibility of $e$ in terms of the operation $z$ can be determined as follows: 1$)$ If $I L_{z}(e)=(s+, d *)$, “*” can be " + " or ",- " then $e$ is accessible. 2) Else e is inaccessible. The following lemma is proved in [18].

Lemma 2. The accessibilities of the nodes in an XML tree can be correctly induced from the ICAM generated by Algorithm 1.

The lookup algorithm for the ICAM in a unit region aims at inducing the label for a requested operation for a requested node, which can be derived by following the steps outlined in the LIR rule. If we store the ICAM as a trie as suggested in [25], i.e., each node is identified by a string which is a prefix of the identifier of its descendants, then the time complexity of this lookup algorithm is $O$ (depth of the requested node in the XML tree $\times \log ($ size of the ICAM)) [25]. In Section 5, we propose a mechanism to enhance the performance of the lookup algorithm.

\subsection{Construction and Lookup for Multiunit Regions}

In the CAM for a single operation with multiple unit regions, there exists some accessible node with an inaccessible parent. This node is called a marker node, and the inaccessible parent is called an interregion terminal node, IRT for short [25]. In an ICAM, this situation also exists. We extend the definition of marker nodes. A marker node for an operation $x$ refers to a node at which $x$ is permitted but denied at its parent. We call an XML tree with at least one marker node a Multiunit Region. In this case, the construction and lookup algorithm need some modification.

According to the construction algorithm of multiunit regions in [25], the parent of a marker node is never labeled in the CAM. That means the label $(s-, d+)$ is not allowed in the CAM. We make similar restriction by enforcing Lemma 1 on the labels of the ICAM.

The basic idea of constructing a CAM in multiunit regions is to decompose the tree into several unit regions and reduce the tree in each individual region. Then, we combine the labels in each region to form a CAM, in which the marker nodes are marked. We adopt a similar idea, with some augmentation:

1. Label the IRT as $(s-, d-)$. As a result, the Label Merging Rule (LMR) can still be applied in multiunit region case.

2. The marker nodes should be identified in the ICAM with the proper operation identifier. Even if its label is upward redundant, it will not be removed.

As for the lookup algorithm, there are details that are worth noting. Suppose we look for the accessibility of node $e$ for operation $z$, and $e$ is not labeled in the ICAM, nor is it a marker node. Suppose $f$ is the nearest labeled ancestor, if any, of $e$ in the ICAM, and $L(f)=(s x, d y)$ :

1. If $f$ is an IRT for operation $z, L_{z}(f)=(s-, d-)$ as we have mentioned above. Then, after merging the labels, we have $y<z$ and $x<z$. Thus, $I L_{z}(e)=(s-, d-)$. On the other hand, $L_{z}(e)=$ $(s-, d-)$ as it is a nonmarker descendant of an 
IRT for operation $z$. Hence, the accessibility of $e$ can be correctly induced in this special case.

2. If $e$ has no labeled ancestor, or $L(f)$ satisfies $x \geq z$ and $\neg(y \geq z)$, we have to check whether $e$ has a nonmarker nearest labeled descendant with label $(s m, d n)$ and $m \geq z$. If it does, $e$ is accessible, otherwise, $e$ is inaccessible in terms of operation $z$.

3. If $f$ is the nearest labeled ancestor of $e$ and $y \geq z$, we have to further check whether $e$ is an descendant of an IRT for operation $z$. That is, among the children of $f$ in the ICAM, we look for $d$, which is a marker for operation $z$ and whose parent is the ancestor-or-self of $e$. If there is such a $d$, meaning $e$ is a descendant of an IRT for $z$, then $e$ is inaccessible, otherwise it is accessible in terms of operation $z$.

Items 2 and 3 are mentioned in [25], while item 1 is special for ICAM because an IRT is not labeled and cannot be the nearest labeled ancestor of any node in a CAM.

Using similar data representation as in [25], the time complexity of this algorithm is $O$ (depth of the requested node in XML tree $\times$ logarithm (size of ICAM)).

\section{EfFicient LoOKup}

In this section, we propose an efficient way of lookup, which can be applied to both CAMs and ICAMs. By adopting a proper numbering scheme for the XML tree and creating indexes for both the XML tree and the CAM/ICAM, our lookup algorithm is more efficient than that of [25].

\subsection{Numbering Scheme}

One of the keys of the accessibility lookup is the determination of ancestor-descendant relationship, which can be assisted by a proper node identifier scheme. There are several methods to identify nodes in an XML tree. Yu et al. [25] adopts a scheme similar to the Dewey notation. Hence, the ancestor-descendant relation determination involves comparing two strings, which is time consuming. We apply a numbering scheme to represent nodes in an XML tree and the ICAM. To every node in the XML tree we assign a 5-tuple (nodeinfo) and store it as an attribute of that node in the XML tree. The 5-tuple is (level_num, level_order, parent_order, pre_order, range), where:

1. level_num: the level of the node. The root belongs to level 0.

2. level_order: the left to right order of the node on its level. Also begins with 0 .

3. parent_order: the preorder number of the direct parent of the node.

4. pre_order: the preorder number of the node.

5. range: the number of descendants of the node.

Fig. 1 shows an example XML tree with nodeinfo(we omit some nodeinfos due to space limitations). Among these five numbers, level_num, pre_order, and range are similar to those of the numbering scheme proposed by [16]. ${ }^{6}$ Using these three numbers, the structural relation between two nodes can be determined in constant time according to a lemma in [16]. In our case, given two nodes, $x$ and $y$, with

6. It is easy to extend to a duration numbering scheme [16] with an extended preorder to accommodate future insertions. Since we do not discuss the update of CAM and ICAM, using preorder is convenient for illustrating our idea.

\begin{tabular}{|c|c|c|c|}
\hline & Node_Info & Access_Label & ptrChildren \\
\hline 0 & $(0,0,0,0,30)$ & $(\mathrm{sw}, \mathrm{dn})$ & $(1,2,3,4,7)$ \\
\hline 1 & $(2,0,1,2,2)$ & $(\mathrm{sw}, \mathrm{dn})$ & NULL \\
\hline 2 & $(2,1,1,5,3)$ & $(\mathrm{sw}, \mathrm{dr})$ & NULL \\
\hline 3 & $(2,2,1,9,2)$ & $(\mathrm{sw}, \mathrm{dr})$ & NULL \\
\hline 4 & $(1,1,0,12,8)$ & $(\mathrm{sw}, \mathrm{dn})$ & $(5,6)$ \\
\hline 5 & $(2,3,12,13,2)$ & $(\mathrm{sr}, \mathrm{dr})$ & NULL \\
\hline 6 & $(2,4,12,16,2)$ & $(\mathrm{sr}, \mathrm{dn})$ & NULL \\
\hline 7 & $(1,2,0,21,6)$ & $(\mathrm{sr}, \mathrm{dn})$ & NULL \\
\hline
\end{tabular}

Fig. 5. The logical table structure of an ICAM.

xinfo and yinfo as their 5-tuple node info, respectively, then $x$ is $y^{\prime}$ s ancestor iff

$$
\begin{aligned}
x i n f o . p r e \_o r d e r & <y i n f o . p r e \_o r d e r \leq x i n f o . p r e \_o r d e r \\
& +x i n f o . r a n g e .
\end{aligned}
$$

Also, if $x$ info.level_number $=$ yinfo.level_number -1 , then $x$ is the parent of $y$.

\subsection{Storage Mechanism of ICAM}

Suppose an ICAM is created for a user or user-group for the original XML tree. It is stored as a table. Here, we use an example to explain the mechanism. Suppose we want to create an accessibility map for user A for an XML tree T. The XML tree as well as the accessibility information is shown in Fig. 1 and the ICAM of that tree is shown in Fig. 2c. Fig. 5 shows a table for the logical structure of the ICAM and also the example data. Each row in the table corresponds to a node in the ICAM. It has three elements: Node_Info is the assigned 5-tuple of the node, Access_Label records the accessibility information, i.e., the label in the ICAM, while ptrChildren contains the pointers to its direct children in the ICAM. (In practice, in order to avoid variable length arrays, two pointers can be used instead of a list of child pointers, one of which points to the first child and the other to the next sibling.)

\subsection{Indexes on the XML Tree and the ICAM}

Indexes are created to quickly locate nodes in an XML tree and the ICAM:

1. We create an index array on the source XML tree. The method flattens the XML tree by traversing it in preorder, and an array (Index_Arr) is built. The $i$ th element of the array has a pointer pointing to the $i$ th node in the XML tree in preorder. Thus, as long as the preorder of a node is known, we can locate the node and get its nodeinfo in constant time. Using the parent_order, combined with the index array of the XML tree, we can traverse from the node to the root of the XML tree in a time proportional to the depth of the node.

2. A 2-layer hash index ${ }^{7}$ is created on the two elements of nodeinfo, level_num, and level_order, to quickly locate entries in the ICAM. We first hash the nodes on the same level of the XML tree into one bucket.

7. Note that we could have hashed on one layer only, e.g., if we only hash on the preorder, then there is no need of the level-order. But, in the case when ICAM is much smaller than the XML tree, there may be a lot of empty buckets using a one layer hash index. By using a 2-layer hash index, we can adjust the number of entries of the second layer, which will save some space. 


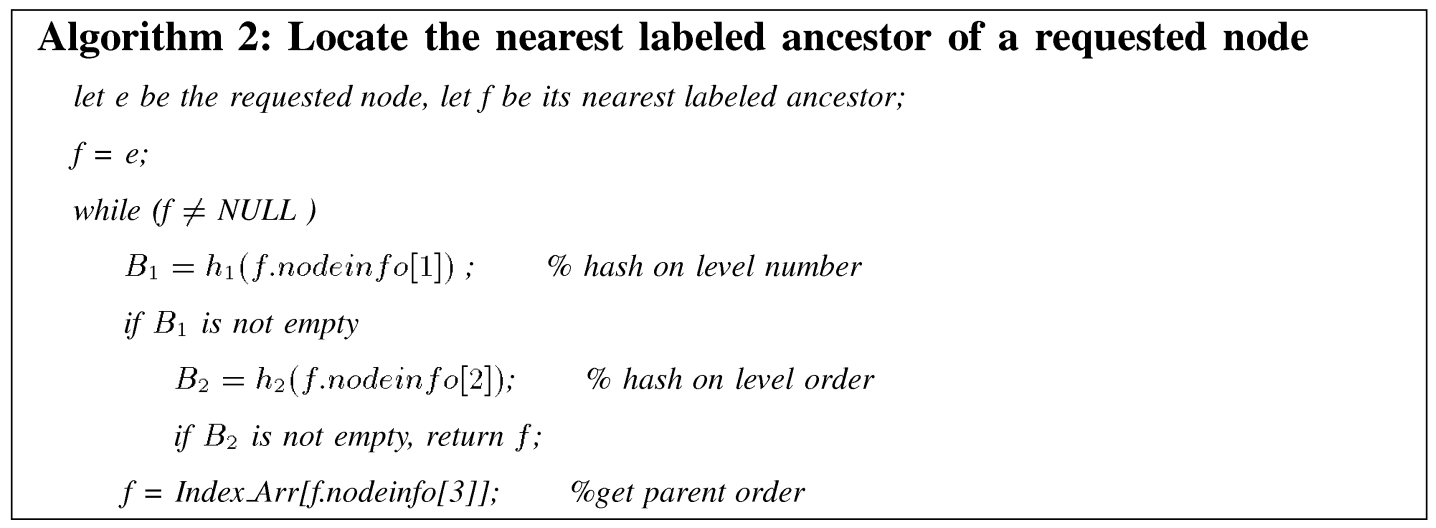

Fig. 6. Locate the nearest labeled ancestor of a requested node.

The level of a node can be obtained by the first element of its 5-tuple nodeinfo. If $h_{1}$ is the hashing function, we have:

$$
\text { FirstLayerBucketNumber }(x)=h_{1}(x . n o d e i n f o[1]) \text {. }
$$

Let $x$ be a node in the ICAM with the highest level_num. Then, the total number of buckets of the first layer of hashing is x.nodeinfo[1] +1 , which is equal to Maximum_ICAM_Level. For example, for the ICAM stored in Fig. 5, it is 3.

Then in each bucket, we further hash the node according to its order among its siblings. The second layer hash function $h_{2}$ gives

\section{SecondLayerBucketNumber $(x)=h_{2}$ (x.nodeinfo[2]).}

The hash table allows a constant average lookup time given sufficient storage space. There is a trade off between speed and space. We can adjust the hash function to reduce the number of empty buckets, while maintaining efficient lookup time. Assisted by the above two indexes, the nearest labeled ancestor of a requested node can be located in time proportional to the depth of the requested node. Fig. 6 describes the procedure.

\subsection{Storing the "Cover" Relation in an Operation Hierarchy}

A "cover" matrix, $M$, is created based on the operation hierarchy in order to determine the "cover" relation between two operations. It is an $n \times n$ matrix, where $n$ is the number of operations, including atomic and composite operations. $M[i, j]=1$ iff operation $i$ covers $j$. In this way, the "cover" relation between two operations can be determined in constant time.

Next, we will discuss how to determine a node's accessibility efficiently.

\subsection{Lookup of the Accessibility Information}

In this section, we explain our lookup algorithm. The key of determining a given node's accessibility is to compute its induced label. Here, we use ICAMs as an example, but note that our algorithm and the following discussion are also applicable to CAMs. The first step of computing the induced label is to locate the nearest labeled ancestor, which is described in Fig. 6. Then, $L I R$ is followed. We give an example to illustrate the procedure.
Example 5. Suppose a request arrives, say an operation $w$ at node $T$ in the XML tree (Fig. 1). We determine its accessibility in the following way:

1. If the nodeinfo of the node is stored with the node in the XML tree, then we can get the nodeinfo of the requested node (i.e., $T$ ) directly. Let T.nodeinfo $=(2,5,12,19,1)$.

2. Calculate the first layer hash function to get to the first layer bucket. We find that

$$
2 \leq \text { Maximum_ICAM_Level. }
$$

That means that $T$ may be labeled in ICAM. Further, hash it by its level_order, that is 5 . We find out that it is not labeled in the ICAM, for the number 5 bucket in the second layer is empty.

3. Get the parent of node $T$ by T.nodeinfo[3], the preorder number of $T^{\prime}$ s parent. Given this number, we can make use of the index array (Index_Arr) to locate the parent in constant time and obtain the nodeinfo of $T^{\prime}$ s parent, i.e., $M$. And $M$. nodeinfo $=(1,1,0,12,8)$.

4. By calculating the value of the first layer hash function for $M$, we find that it should appear in the first bucket of the hash table. Further, calculate the second layer hash function to locate $M$ in the first bucket. Thus, we get the entry of $M$ in the ICAM table. $L(M)=(s w, d n)$. As $\neg(n \geq w)$, we need to find out whether $T$ has a labeled descendant.

5. From the ptrChildren field of the $M$ entry, we know that $M$ has two direct children in the ICAM, that is, $N$ and $Q . N$.nodeinfo $=(2,3,12,13,2)$ and Q.nodeinfo $=(2,4,12,16,2)$. Both of them are not the descendants of $T$, for 13 and 16 do not fall in the range of $(19,20]$. We know that if $T$ were an ancestor of $N$, it should satisfy the inequality:

$$
\begin{aligned}
\text { T.nodeinfo.pre_order } & <\text { N.nodeinfo.pre_order } \\
& \leq \text { T.nodeinfo.pre_order } \\
& + \text { T.nodeinfo.range. }
\end{aligned}
$$

So does $Q$. Therefore, we deduce that $T$ has no accessible descendants. Hence by $L I R$, $I L_{w}(T)=(s-, d-)$. So, we know that $T$ is inaccessible in terms of operation $w$. 


\subsection{Time Complexity}

We state in this section the results concerning the time complexity of the algorithm for looking up a node in the ICAM.

1. The time needed to assign the nodeinfo to nodes. This is done only once for every XML tree. The time is proportional to the number of nodes in the XML tree.

2. The time to construct the ICAM. The time is proportional to the XML tree size (number of nodes in the XML tree [25]).

3. The lookup time. Since the requests may be frequent and the users may not want to wait a long time for the results, this is the time we try to reduce. There are two components: 1) The time needed for looking up a requested node's nearest labeled ancestor in the ICAM. It is proportional to the number of levels in the XML tree between the requested node and its nearest labeled ancestor in the ICAM. 2) For a CAM, the label for an operation is obtained directly. While for an ICAM, the label for an operation is induced from the ICAM as well as from the cover relation between the two operations. But based on the cover matrix for the operation hierarchy, the cover relation between two operations can be determined in constant time, implying the label for an operation can be induced in constant time given the label in the ICAM. If the label of the nearest labeled ancestor is $(s x, d y)$ and $x \geq z$ but $\neg(y \geq z)$, or the requested node has no labeled ancestor, we need to determine if the requested node has any accessible descendants. So, we have to search in the children of this labeled ancestor in the ICAM, or the roots of the ICAM if no labeled ancestor exists, to see if there exist any descendants of the requested node with label $(s m, d n)$ and $m \geq z$. It will take time proportional to the fanout of the requested node's nearest labeled ancestor in the ICAM.

Lemma 3. The overall time of lookup is $O$ (depth of the requested node in the XML tree + the maximum fanout of CAM/ ICAM).

But, if we sort the children of each node in the ICAM by their preorder numbers, the time complexity will be reduced to be the logarithm of the fanout. This is because we can determine the range of preorder numbers for descendants of the requested node and we can search the sorted list by binary search in logarithmic time.

Lemma 4. If the child-pointers of each ICAM node are sorted, the overall time of lookup is $O$ (depth of the requested node in the $X M L$ tree + $\log ($ the maximum fanout of CAM/ICAM)).

Compared with the time complexity of [25], which is $O$ (depth of the requested node in XML tree $\times \log (C A M / I C A M$ size)), the proposed algorithm has a much lower complexity.

\subsection{Space Requirement}

We now discuss how we measure the space for CAM/ ICAM. It includes the space for the CAM/ICAM, its hash index, and the index on the original XML tree.
The space needed for CAM/ICAM is the number of nodes in CAM/ICAM times the number of bits needed for each node, which includes the node identifier, the label, and the necessary pointers. For the node identifier, if we adopt the 5-tuple nodeinfo as the node identifier, 4 bytes for each number, then a total of 20 bytes are needed. ${ }^{8}$

Pointers are needed to link the nodes in the CAM/ ICAM. In practice, for each node two pointers are maintained, one linking to its first child, the other to its right sibling. Assuming that each pointer requires 4 bytes, then 8 bytes are needed for storing the pointers of each node in the CAM/ICAM.

As for the label, in a CAM, three bits are enough for a label, one for the " $\mathrm{s}$ " part, one for the " $\mathrm{d}$ " part, and the third one for a marker. While in an ICAM, the number of the bits needed for each part of a label depends on the number of operations. For example, in the hierarchy in Fig. $3 d$, there are eight operations, $R, U, D, I, U D, U I, D I, U D I$. Thus, three bits are needed to distinguish them. The "s" and " $\mathrm{d}$ " parts require three bits each. (In general, for $n$ operations, we need $\log n$ bits each.) We also need to indicate whether a node is a marker for an operation or not. One marker bit has to be assigned to each atomic operation, i.e., four bits are needed in total.

In other words, if we adopt 5-tuple nodeinfo for both CAM and ICAM. The number of the bits needed for each node in CAM/ICAM is given by the following equations:

$$
C A M: \quad(20 \times 8)+(8 \times 8)+3=227 \text { (bit) },
$$

$$
\text { ICAM : } \quad(20 \times 8)+(8 \times 8)+(3 \times 2)+4=234 \text { (bit). }
$$

The size of the hash index is affected by two factors: the size of the CAM/ICAM and the distribution of nodes in the CAM/ICAM. The number of nonempty buckets is bounded by the level of ICAM times the maximum number of the nodes on one level. Note that for the CAM, each hash index has be to created for each operation. But, the ICAM requires only one hash index. The index array of the original XML tree, which is stored outside the XML tree, takes the space proportional to the number of nodes in the XML tree. These indexes are used to speed up the lookup. The same algorithm can also be applied to the CAM.

\section{EXTENSION OF ICAM}

To further reduce lookup time, we can add nodes into the ICAM. After constructing the ICAM, do one more traversal of the ICAM. If we observe that the XML level between the parent and the child in the ICAM is bigger than a certain threshold, we can add more nodes on this path simply by reinserting the deleted nodes. We call the resulting ICAM as Extended ICAM (EICAM). The size of EICAM is controlled by the value of the threshold.

8. If we use the method of [25], the length of a node identifier depends on the depth of the node. Take a full binary XML tree as an example, the average length of the identifier can be computed by $\frac{1}{2^{k}-1} \sum_{i=1}^{k} i \times 2^{i-1}$, where $k$ denotes the level of the tree beginning from 1. For a 18-level binary tree, the average length of the node identifier is 17 . Using 4 bytes for each symbol, then, on average, 68 bytes are needed to identify a node, which is much longer. 
Lemma 5. EICAM represents the same accessibility as the original ICAM.

Proof. Here, we prove that for any $C A M_{z}$, labels of the other nodes can still be correctly induced after adding back an induced label $l$ (the resulting CAM is denoted by $C A M_{z}^{\prime}$ ). For we have proven in Theorem 3 that after the label merging, the labels of the nodes for an operation can be correctly induced.

Thus, given an unlabeled node $e$, we prove that the induced label $L_{z}(e)$ in $C A M_{z}$ equals to the induced label $L_{z}^{\prime}(e)$ in $C A M_{z}^{\prime}$. Let $f$ and $g$ denote the nearest labeled ancestor and descendant of $e$ in $C A M_{z}$, respectively, if any. Adding $l$ to $C A M_{z}$ can affect the induced label of $e$ only if $l$ becomes $e^{\prime}$ s nearest labeled ancestor or descendant in $C A M_{z}^{\prime}$.

Case 1. $l$ is the nearest labeled ancestor of $e$ in $C A M_{z}^{\prime}$. If $L_{z}(f)=(s+, d+)$ (or $(s-, d-)$, respectively), according to Definition 3, $L_{z}(l)=L_{z}(e)=(s+, d+)$ (or $(s-, d-)$, respectively). On the other hand, in $C A M_{z}^{\prime}$, since $L_{z}^{\prime}(l)=$ $(s+, d+)$ (or $(s-, d-)$, respectively), $L_{z}^{\prime}(e)=(s+, d+)$ (or $(s-, d-)$, respectively), equals to $L_{z}(e)$.

If $L_{z}(f)=(s+, d-)$ or no $f$ exists, $L_{z}(e)$ and $L_{z}(l)$ can be $(s+, d-)$ or $(s-, d-)$, depending on whether $e$ and $l$ have an accessible descendant. If there is such $g$ with label $(s+, d *), L_{z}(e)=L_{z}(l)=(s+, d-)=L_{z}^{\prime}(l)$. And, it is obvious that $L_{z}^{\prime}(e)$ also equals $(s+, d-)$ in this case. If there is no such $g, L_{z}(e)=L_{z}(l)=(s-, d-)=L_{z}^{\prime}(l)$. Then, in $C A M_{z}^{\prime}$, since $L_{z}^{\prime}(l)=(s-, d-), L_{z}^{\prime}(e)=(s-, d-)=L_{z}(e)$.

Case 2. $l$ is the nearest labeled descendant of $e$ in $C A M_{z}^{\prime}$

If $L_{z}(f)=(s+, d+)$ (or $(s-, d-)$, respectively), $L_{z}(e)=$ $L_{z}^{\prime}(e)=(s+, d+)$ (or $(s-, d-)$, respectively) irrespective of its labeled descendants.

If $L_{z}(f)=(s+, d-)$ or no such $f$. If $e$ has a nearest labeled descendant, say $g$, in $C A M_{z}$. It should also be the nearest labeled descendant of $l$, otherwise, it should be the label of $g$ that we have reinserted, instead of $l$. Thus, if $L_{z}(g)=(s+, d *), L_{z}(e)=L_{z}(l)=(s+, d-)=L_{z}^{\prime}(l)$. It is obvious that in this case, $L_{z}^{\prime}(e)=(s+, d-)$, too. If $e$ has no such $g$ in $C A M_{z}, l$ does not either. Thus,

$$
L_{z}(e)=L_{z}(l)=(s-, d-) .
$$

Then in $C A M_{z}^{\prime}, L_{z}^{\prime}(e)=(s-, d-)$.

Therefore, the label of $e$ can still be correctly induced in $C A M_{z}^{\prime}$.

In this way, we can further reduce the time for looking up the nearest labeled ancestor to the value of threshold. It is the trade off between the space and the lookup time.

Lemma 6. The lookup time of the EICAM is independent of the depth of the node in the XML tree.

Proof. As discussed in Section 5.6, looking up a node in an ICAM involves locating the nearest labeled ancestor and descendants. In the ICAM, locating the nearest labeled ancestor is linear to the depth of the requested node. While in the EICAM, by adding back an induced label, the number of the nodes on the path from the nearest labeled ancestor of the requested node to the requested node in the XML tree is upper bounded by the threshold, i.e., irrespective of the depth of the node. While the time complexity of looking for a nearest label descendant is still $O(\log$ (maximum fanout of EICAM)). In other words, looking up a node in the EICAM is independent of the depth of the node in the XML tree.

\section{EXPERIMENTAL RESULTS}

We have conducted experiments to show how the use of ICAM can help reduce the storage size of the accessibility maps and verify the efficiency of our lookup algorithm. All the experiments are conducted on a SUN Enterprise E4500, running Solaris 7 and having 8G RAM. The synthetic XML data is generated by the IBM XMLGenerator [9]. There are several parameters in the XMLGenerator to control the size, the depth, and the breadth (i.e., the average number of nodes on one level) of the input XML tree. As a result, we can verify our algorithms on the XML trees of different sizes and shapes. The real data is the accessibility control information of the Unix file system of a big university. We report here the experiments we conducted to test space efficiency as well as the construction and lookup time complexities of CAM, ICAM, and the full materialized map (FMM). The full materialized map is the accessibility map containing all the accessible nodes and no inaccessible node while maintaining the structural relationship among the nodes.

We adopt two mechanisms for node identity, one is the trie mechanism, as is used for CAM in [25]; the other is the numbering scheme we described in Section 5.1. We refer to these two kinds of CAMs as CAM_T (T stands for TRIE), and CAM_N (N stands for numbering scheme), respectively. Similarly, we distinguish between FMM_T and FMM_N. We adopt the operation hierarchy in Fig. 3d in the experiments and use the encoding method to represent the operations. Note that only the CAMs for the atomic operations are built for CAM_N, CAM_T, or ICAM.

\subsection{Experiments on Space Efficiency}

In the experiments on space efficiency, we use the same set of synthetic data as in [25]. The parameters used in the experiments are as follows: the total number of nodes in the XML tree is 16,811 , fanout is varied from 1 to 60 and has an average value of 2 (not including the leaves), and the average depth of the tree is 8 . We also adopt parameters from [25] to control the accessibility ratio and access locality. They call a node friendly if the nodes in the subtree rooted at that node have a high probability of being accessible. The subtree rooted at this root is called a friendly area. Otherwise, the node is called nonfriendly and the subtree rooted at the node is a nonfriendly area. The related parameters are:

1. af/anf: The access probability of a node in a friendly/ nonfriendly area. In the experiments, we set these two parameters to be $\mathbf{9 8}$ percent and $\mathbf{2}$ percent, respectively, as in [25], to obtain high structural accessibility locality.

2. fr/rr: The friendly ratio/reverse Ratio. It is the probability that a node is a friendly/nonfriendly node given that its parent is a nonfriendly/friendly node. We have to keep fr small in order to achieve high access locality. In the experiments, we set it to be 5 percent. While $r r$ is varied to control the accessibility ratio. In the experiment, we vary $r r$ from $\mathbf{0}$ percent to $\mathbf{1 0 0}$ percent. These settings are also followed in [25]. 


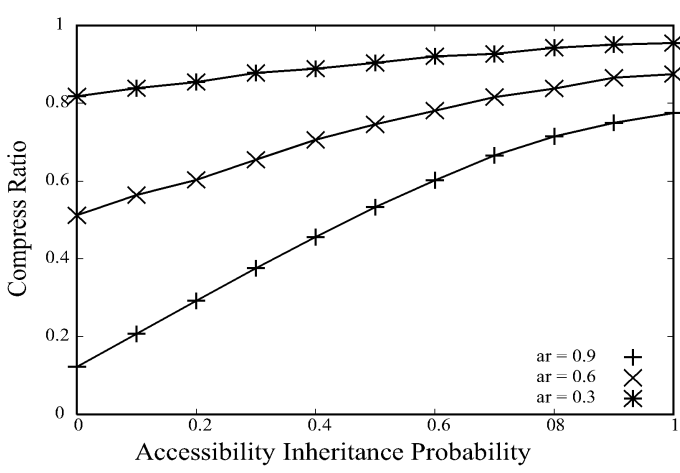

(a)

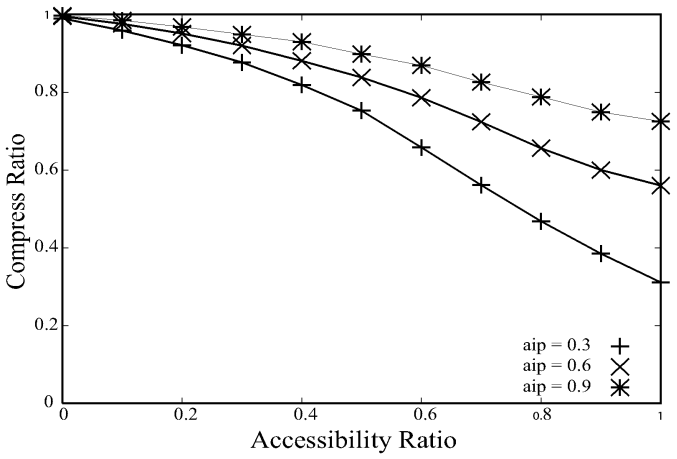

(b)

Fig. 7. Results about Compress Ratio.

3. ar: The accessibility ratio (ar) is given by $1-r r$. This factor is an indication of the amount of nodes that are accessible in the XML tree.

Besides the above parameters, we adopt another parameter to simulate the accessibility distribution among the nodes. We called it Accessibility Inheritance Probability (aip). It is defined as follows: Given an XML tree and a node $e$ in the tree, for two different operations, $x$ and $y$, where $x>y$, suppose $y$ is permitted at $e$, then aip is a conditional probability that $x$ is also permitted at $e$. In the experiment, we vary aip from $\mathbf{0}$ percent to $\mathbf{1 0 0}$ percent to get various different accessibility similarities among the operations.

We generate the accessibility information of the data as follows: Considering an operation hierarchy in Figs. 3b, 3c, and $3 \mathrm{~d}, a f / a n f, f r / r r$, and $a r$ are to determine whether a node is accessible for $R$. We call the nodes that are accessible at least for $R$ as accessible nodes. Then, among all the accessible nodes, aip is used to control the accessibility probability of the other operations in the operation hierarchy. Hence, we keep fr low to achieve high access locality for $R$. For the other operations, $U, I$, and $D$, their access probabilities are controlled by aip, and the access locality is not guaranteed.

The hierarchy in Fig. 3a is for the experiments on the Unix file system data only. As the accessibility information is already there, we do not need the above parameters to generate the accessibility information.

In the experiments, we use the same definition of Compress Ratio as that in [25].

Definition 6 (Compress Ratio). The Compress Ratio (CR) of an ICAM is defined by the following equation:

$$
\mathrm{CR}=\frac{\text { Size of }(I C A M)}{\text { Total number of accessible nodes }} .
$$

The size of a CAM or an ICAM is given by the number of nodes in the CAM and ICAM, respectively. We also define the term Gain Ratio, which is used to measure the space saved by combining all CAMs into one ICAM.

Definition 7 (Gain Ratio). The Gain Ratio (GR) of an ICAM integrated from $C A M_{1}, \ldots C A M_{n}$ is defined by the following equation:

$$
\mathrm{GR}=\frac{\sum_{k=1}^{n} \operatorname{BitsOf}\left(C A M_{k}\right)-\operatorname{BitsOf}(I C A M)}{\sum_{k=1}^{n} \operatorname{BitsOf}\left(C A M_{k}\right)} .
$$

The BitsOf() function computes the product of the number of nodes in CAM/ICAM and the number of bits of each node. The latter part is determined by (1) and (2), respectively (see Section 5.7). Since the numbering scheme has obvious advantages, from both complexity analysis and empirical studies in construction time and accessibility lookup (see results in this section), we assume that both CAM and ICAM adopt the 5-tuple nodeinfo as identifiers when calculating the Gain Ratio, i.e., CAM_N is considered here.

Compress ratio and gain ratio are two target measurements in our experiments. We study the influence of various parameters on these two ratios. There are several conditions that may affect these two targets: access locality, accessibility ratio, similarities among the CAMs for different operations and the size and shape of the operation hierarchy.

\subsubsection{Compress Ratio}

In this section, we will discuss how the above parameters affect the compress ratio.

Compress Ratio Versus Accessibility Inheritance Probability. Accessibility Ratio (ar) is fixed at 30 percent, 60 percent, and 90 percent, representing small, medium, and high accessibility ratio, respectively. aip is varied from 0 percent to 100 percent. The result is shown in Fig. 7a. From the figure, we observe that $\mathrm{CR}$ increases when aip increases. That is because when aip increases, the number of nodes that are permitted to do the operations on the second layer or above, i.e., operation $U, I$, and $D$, increases, too. That leads to much increase in the number of nodes labeled in the ICAM. Meanwhile, the number of accessible nodes remains unchanged, hence, the compress ratio increases.

Compress Ratio Versus Accessibility Ratio. aip is fixed at 30 percent, 60 percent, and 90 percent, representing small, medium, and high accessibility inheritance probability, respectively. And, ar is varied from 0 percent to 100 percent. The result is shown in Fig. 7b. The result shows that CR decreases with the increasing of $a r$. 


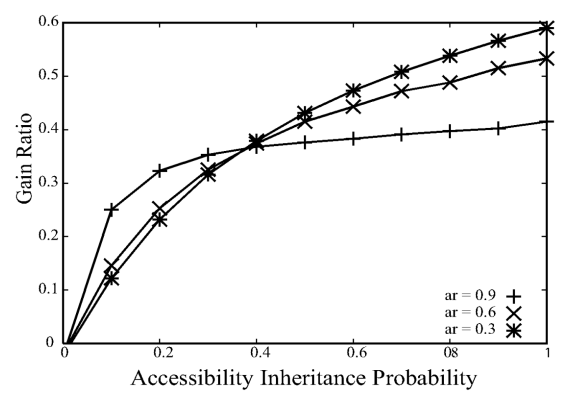

(a)

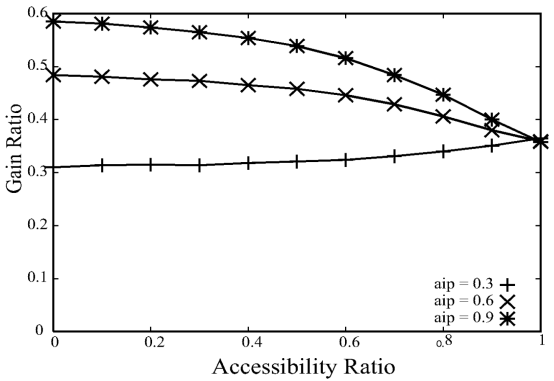

(b)

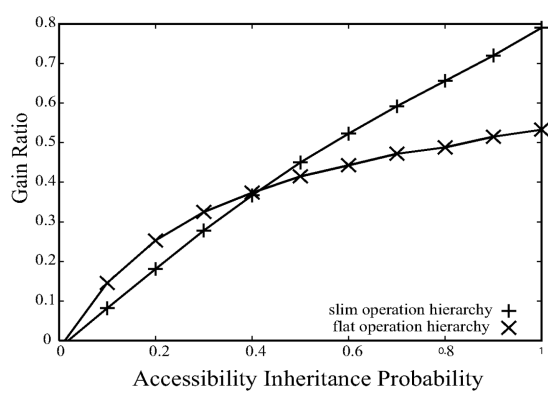

(c)

Fig. 8. Results about gain ratio.

\subsubsection{Gain Ratio}

This section lists the result of the experiments on gain ratio.

Gain Ratio Versus Accessibility Inheritance Probability. ar is fixed at 30 percent, 60 percent, and 90 percent, respectively, representing small, medium, and high accessibility ratio. The value of aip is varied from 0 percent to 100 percent. The result is shown in Fig. 8a. From the figure we observe that GR increases as aip increases. That means the higher the accessibility inheritance probability, the more space is saved by ICAM. The highest gain ratio achieved is near 0.6 , meaning that close to 60 percent of the space is saved.

Gain Ratio Versus Accessibility Ratio. The value of aip is fixed at 30 percent, 60 percent, and 90 percent, representing small, medium, and high accessibility inheritance probability, respectively. $a r$ is varied from 0 percent to 100 percent. The result is shown in Fig. $8 \mathrm{~b}$. The result shows that the gain ratio changes little with the accessibility ratio. That means the accessibility ratio has little effect on the space saved by ICAM.

Gain Ratio Versus the Shape of the Operation Hierarchy. This experiment shows the influence of the shape of operation hierarchy to the gain ratio. Here, we compare the gain ratio when the system supports the operation hierarchy of Figs. $3 b$ and $3 d$, respectively. Due to page limitation, we only list the result in the case of the medium accessibility ratio ( $\mathrm{ar}=60$ percent). As plotted in Fig. 8c, the gain ratio of the slim hierarchy increases faster with aip than that of the flat hierarchy. Because the slimmer the hierarchy, the higher the probability of the overlapping of the CAMs, which leads to greater gain ratio.

\subsection{Construction Time}

Experiments are conducted to compare the construction time of CAM_N, CAM_T, and ICAM. The construction of a CAM or an ICAM includes two processes, constructing and storing. When creating an ICAM for several operations, the merging and storing of labels is done only once for all operations. While to construct several CAMs, the storing process has to be done once for each operation.

In order to maintain the structural relationship among the nodes in the CAM and ICAM, the descendant-ancestor relationship of two nodes has to be determined when linking the nodes, and the efficiency of this step greatly affects the construction time. If the trie scheme is used to encode node identifier, the determination takes much longer time because of string comparison. While the time complexity becomes constant if the numbering scheme is used. That is why the construction time of CAM_T is longer than those of CAM_N and ICAM, as the results of the experiments show.

We conduct the experiments under several situations. But, due to space limitation, we only show the results with the following settings: $a r=60$ percent, $f r=5$ percent, $\mathrm{af}=98$ percent, $\mathrm{anf}=2$ percent, and aip $=60$ percent. In other words, the following results are for the case of medium accessibility ratio, access locality, and accessibility inheritance probability.

XML trees of different sizes are generated by the IBM XMLGenerator. We compare the average construction time of CAM_N, CAM_T, and ICAM for four operations. The results are given in Fig. 9. The results show that the ICAM and CAM_N are constructed more than two times faster than CAM_T. The construction time is linear to the size of the XML tree. Label merging and storing do not cost extra overhead when constructing an ICAM. The experimental results confirm the earlier performance claims.

\subsection{Experiments on Accessibility Lookup}

In this section, we present the experiments and their results to verify the performance claims on the lookup. The same data set as in Section 7.1 is used for these experiments.

The parameters for controlling accessibility assignment are set as in the previous experiments on construction time. First, we apply our numbering scheme and indexes to the CAM (CAM_N) and compare the lookup time of our algorithm with that of the original CAM (CAM_T). The

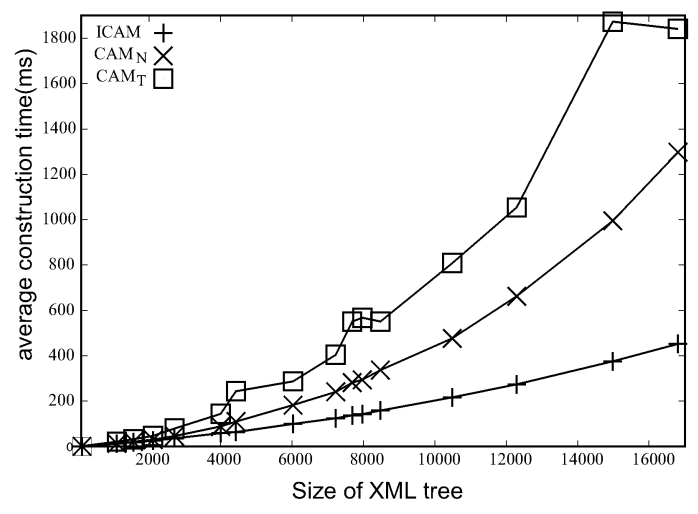

Fig. 9. Construction time for four operations. 


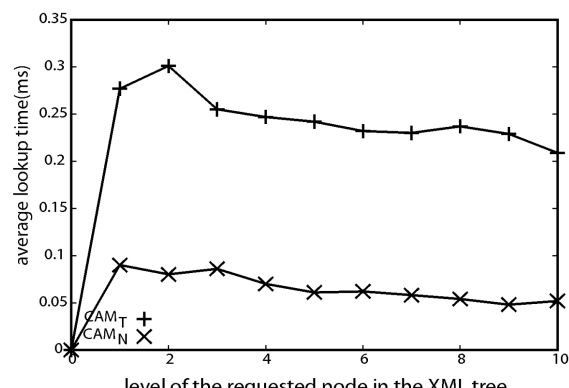

level of the requested node in the XML tree

(a)

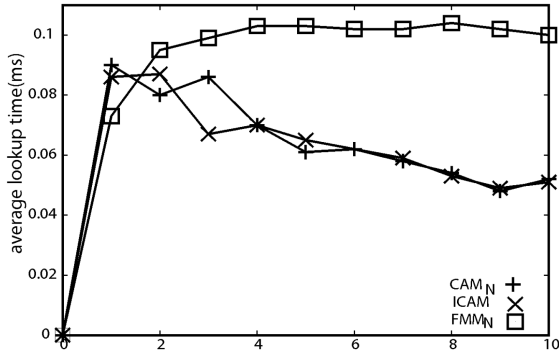

level of the requested node in the XML tree

(b)

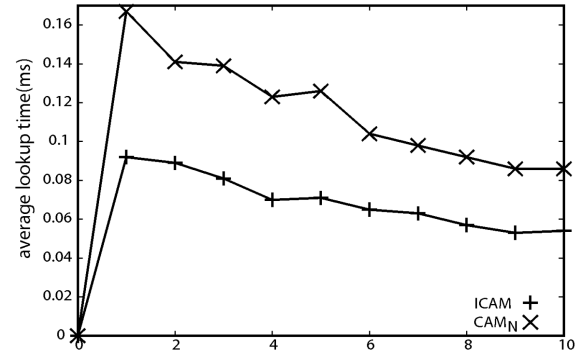

level of the requested node in the XML tree

(c)

Fig. 10. The average lookup time.

result, as shown in Fig. 10a, verifies that our lookup algorithm is much more efficient. As a result, we only consider the case of using numbering scheme in the following experiments.

Then, we examine the effects of integrating multiple operations. We first test the average lookup time when the user asks for only one operation. Fig. 10b shows the average lookup time of each level of an XML tree. The result shows that merging labels in the ICAM does not cost extra time when a user wants to look up only one operation. And, the lookup in both CAM and ICAM are faster than that in FMM_N. Because when looking up in the ICAM/CAM, most searching stops once a labeled ancestor is found. But, the lookup in an FMM usually needs to travel the whole path from the root to the requested node if accessible or to one of its descendants if inaccessible. The average lookup time for the nodes at level one in the ICAM/CAM is larger because there are many more labeled nodes at that level.

The third experiment shows the average lookup time of CAM_N and ICAM for two operations. As illustrated in Fig. 10c, the ICAM is optimal when the user wants to lookup multiple operations, for the nearest labeled ancestor has to be located only once.

\subsection{Experiments on Real Data Set}

We also conduct the experiments on a real data set. The data set is the UNIX file system data, which has the similar structural and accessibility characteristic with the XML data. Our data is obtained from an existing system of the computer science department of the Chinese University of Hong Kong. It has 408,561 nodes and 271 users. The maximum fanout of the file structure is 3,033 and the average is 15 . The maximum depth is 15 and the average is 7. We assume that the three operations, $\operatorname{read}(r)$, write $(w)$, and execute $(x)$, form an operation hierarchy shown in Fig. 3a. We construct the ICAM and CAM_Ns on the data. The average compress ratio of the ICAM is 0.3 and the average gain ratio of ICAM over CAM_N is 20 percent. The gain ratio is not very big. As the file system has high accessibility locality, the compress ratios of the CAMs are good. Hence, the gain of the ICAM over the CAMs is not that much. But, it still saves about 20 percent space. We also compare their construction time and lookup time. The average construction time of the CAM_N for the three operations is about 135.5 seconds, and that for the ICAM is only about 81.7 seconds. Fig. 11 plots the lookup time of two operations in the ICAM and CAMs, respectively. It shows that the lookup in the ICAM is faster than in the CAMs in all the tested cases.

\subsection{Discussion}

The experimental results can be explained as follows:

1. If the identifier of each node in the XML tree is a string extension relative to its parent, that is, the identifier for any node is a prefix of the identifiers for each of its descendants, searching for the nearest labeled descendants or ancestors involves string comparison, which is time consuming. While in our algorithm, with the 5-tuple nodeinfo, the determination of the descendant-ancestor relationship can be done in constant time. This also explains why the construction and the lookup of the CAM_N are faster than those of the CAM_T.

2. The CAMs for different operations are combined into one ICAM. Thus, even when the lookup involves several operations, the nearest labeled ancestor and descendant for the requested node are located only once. While for CAM, this locating process has to be executed multiple times. Hence, ICAM has a better overall lookup performance comparing to CAM.

\section{Conclusion}

In this paper, we study the problem of accessibility control for XML data. We consider the approach of compressed

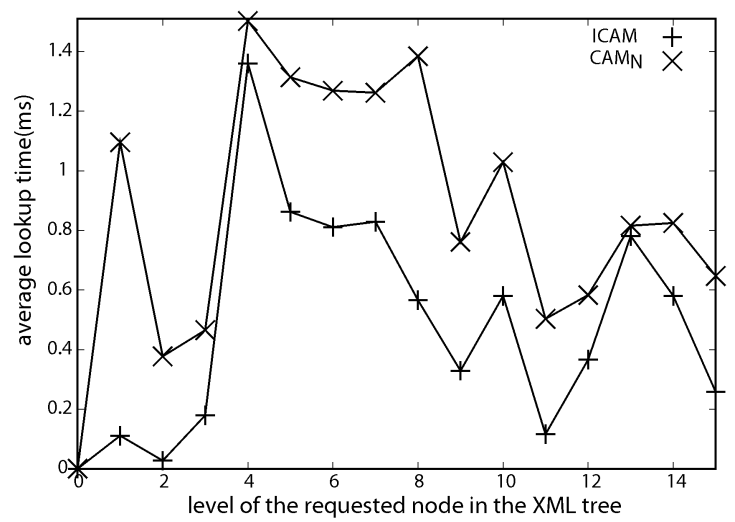

Fig. 11. The average lookup time of the real data. 
accessibility maps (CAM) and propose an algorithm to integrate the optimal compressed accessibility maps for different operation types into an integrated compressed map, ICAM, with a time complexity linear to the data size. The result is a more compressed form of the accessibility map, which also consolidates the information for each user group. With the resulting ICAM, we propose an efficient lookup method to determine the accessibility of a node. This method can greatly improve the complexity of the lookup time provided that the structure of the XML data is not frequently updated. We also propose the possibility of extending the ICAM to EICAM, further reducing the lookup time complexity. The results of the experiments verify the effectiveness of the integration method and the lookup mechanism. From experiments, compared to the method in [25], up to 60 percent of space can be saved by the integration approach and our lookup algorithm can be more than two times faster than that of [25].

One of the future works is to combine the accessibility evaluation with the query evaluation to increase the overall performance of the XML query processing.

\section{ACKNOWLEDGMENTS}

The authors thank Ting $\mathrm{Yu}$ for providing valuable assistance in the experimental work and advice. The authors thank the reviewers for very constructive suggestions and helpful comments. Also they thank Zhixiang Chen for a thorough review of the final draft. This research is supported by the Hong Kong RGC Earmarked Grant UGC REF.CUHK 4179/01E and Hong Kong ITF Grant REF. ITS/069/03.

\section{REFERENCES}

[1] Document Object Model (DOM) Level 1 Specification, version 1.0, W3C recommendation 1, http://www.w3.org/tr/rec-dom-level1, Oct. 1998.

[2] B. Kolman and R.C. Busby, Discrete Mathematical Structures for Computer Science. Prentice-Hall Int'l Editions, second ed. 1987.

[3] E. Bertino, S. Castano, E. Ferrari, and M. Mesiti, "Controlled Access and Dissemination of XML Documents," Proc. Second Int'l Workshop Web Information and Data Management, Nov. 1999.

[4] E. Bertino and E. Ferrari, "Secure and Selective Dissemination of XML Documents," ACM Trans. Information and System Security, vol. 5, no. 3, pp. 290-331, Aug. 2002.

[5] E. Bertino, P. Samarati, and S. Jajodia, "An Extended Authorization Model for Relational Databases," IEEE Trans. Knowledge and Data Eng., vol. 9, no. 1, pp. 85-101, Jan./Feb. 1997.

[6] P. Bird, "Implementing Low Level Access Control with DB2 UDB," The IDUG Solutions J., vol. 7, no. 3, 2000.

[7] E. Damiani, S. De Capitani di Vimercati, S. Paraboschi, and P. Samarati, "Securing XML Documents," Proc. Int'l Conf. Extending Database Technology, pp. 121-135, Mar. 2000.

[8] E. Damiani, S. De Capitani di Vimercati, S. Paraboschi, and P. Samarati, "A Fine-Grained Access Control System for XML Documents," ACM Trans. Information and System Security, vol. 5, pp. 169-202, May 2002.

[9] A.L. Diaz and D. Lovell XML Generator, www.alphaworks.ibm. com/tech/xmlgenerator/, Sept. 1999.

[10] R. Fagin, "On an Authorization Mechanism," ACM Trans. Database Systems, vol. 3, pp. 310-319, Sept. 1978.

[11] W. Fan, C. Chan, and M. Garofalakis, "Secure XML Querying with Security Views," Proc. ACM Int'l Conf. Management of Data, pp. 587$598,2004$.

[12] I. Fundulaki and M. Marx, "Specifying Access Control Policies for XML Documents with XPath," Proc. ACM Symp. Access Control Models and Technologies, pp. 61-69, 2004.
[13] N. Gal-Oz, E. Gudes, and E.B. Fernandez, "A Model of Methods Access Authorization in Object-Oriented Databases," Proc. Very Large Data Bases Conf., 1993.

[14] P. Griffiths and B. Wade, "An Authorization Mechanism for a Relational Database System," ACM Trans. Database Systems, vol. 1, pp. 242-255, Sept. 1976.

[15] M. Kudo and S. Hada, "XML Document Security Based on Provisional Authorization," Proc. ACM Conf. Computer and Comm. Security, pp. 87-96, Nov. 2000.

[16] Q. Li and B. Moon, "Indexing and Querying XML Data for Regular Path Expressions.," Proc. Very Large Data Bases Conf., 2001.

[17] M. Jiang and A. Fu, "Efficient Accessibility Lookup for XML," Proc. Int'l Conf. Databases and Applications (DBA2003), Feb. 2003.

[18] M. Jiang and A. Fu, "Integration and Efficient Lookup of Compressed XML Accessibility Maps," Technical Report CS-TR2005-1, Dept. Computer Science and Eng., Chinese Univ. of Hong Kong, 2005.

[19] M. Murata, A. Tozawa, M. Kudo, and S. Hada, "XML Access Control Using Static Analysis," Proc. ACM Conf. Computer and Comm. Security, pp. 73-84, 2003.

[20] F. Rabitti, E. Bertino, W. Kim, and D. Woelk, "A Model of Authorization for Next-Generation Database Systems," ACM Trans. Database Systems, vol. 16, pp. 88-131, Mar. 1991.

[21] F. Rabitti, D. Woelk, and W. Kim, "A Model of Authorization for Object-Oriented and Semantic Databases," Proc. Int'l Conf. Extending Database Technology, Mar. 1988.

[22] D. Raphaely et al., "Establishing Security Policies," Oracle8i Application Developer's Guide-Fundamentals Release 8.1.5, chapter 12, Feb. 1999.

[23] T. Cormen, C. Leiserson, R. Rivest, and C. Stein, Introduction to Algorithms. The MIT Press, second ed., 2002.

[24] M. Winslett, K. Smith, and X. Qian, "Formal Query Languages for Secure Relational Databases," ACM Trans. Database Systems, vol. 19, pp. 626-662, Dec. 1994.

[25] T. Yu, D. Srivastava, L.V.S. Lakshmanan, and H.V. Jagadish, "Compressed Accessibility Map: Efficient Access Control for XML," Proc. Very Large Data Bases Conf., pp. 478-489, 2002.

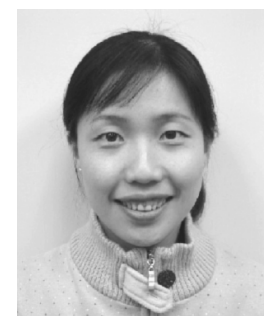

Mingfei Jiang received the BSc and MPhil degrees in computer science from Southeast University of China in 1999 and 2002, respectively. She is now a $\mathrm{PhD}$ candidate in the Department of Computer Science and Engineering at the Chinese University of Hong Kong. Her research interests include XML security, XML index, and XML query.

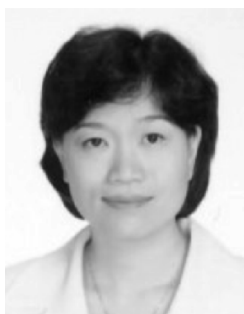

Ada Wai-Chee Fu received the BSc degree in computer science from the Chinese University of Hong Kong in 1983, and both the MSc and PhD degrees in computer science from Simon Fraser University, Canada, in 1986 and 1990, respectively. She worked at Bell Northern Research in Ottawa, Canada, from 1989 to 1993 on a widearea distributed database project before joining the Chinese University of Hong Kong in 1993. Her research interests include issues in XML data, time series databases, data mining, content-based retrieval in multimedia databases, and parallel and distributed systems. She is a member of the IEEE.

For more information on this or any other computing topic, please visit our Digital Library at www.computer.org/publications/dlib. 